\title{
Comportamientos incumplidores: contextos y posibles tratamientos políticos
}

\author{
Cecilia Güemes \\ Isabel Wences Simon \\ Universidad Carlos III de Madrid \\ cecilia.guemes@gmail.com; iwences@polsoc.uc3m.es
}

\section{Resumen}

El presente trabajo tiene dos objetivos. El primero consiste en sistematizar contribuciones analíticas enfocadas a interpretar el incumplimiento normativo, englobándolas en cuatro grandes perspectivas: a) la ineficiencia del gobierno; b) un orden o institucionalidad informal o paralela; c) el umbral de deshonestidad; y, d) la falta de sincronía entre motivos (morales, éticos y legales) e incentivos. El segundo se centra en esbozar tres estrategias políticas novedosas que puedan ayudar a combatir el incumplimiento, especialmente en América Latina. Por un lado, las nudges (intervenciones en la arquitectura de decisión); por el otro, el enfoque think (interiorizar distintas normas sociales); y, finalmente, el desafío de construir nuevas identidades colectivas.

Palabras clave: comportamientos incumplidores; cultura de la legalidad; nudge; enfoque think; teoría de la identidad social

* El presente texto se ha desarrollado en el marco del proyecto de investigación: Programa Interuniversitario en Cultura de la Legalidad (NEW TRUST-CM) de la Comunidad de Madrid. Referencia: S2015/HUM-3466. 
Abstract. Non-compliant behavior: Context and possible political treatment

The present paper has two goals. The first is to sum up some analytical contributions to interpret normative non-compliance which put the focus on: a) government inefficiency; b) an informal or parallel order; c) the threshold of dishonesty; and d) a lack of synchronicity between motives (moral, ethical and legal) and incentives. The second is to outline new political strategies that could be useful to combat non-compliance, primarily in Latin America: nudges (interventions in the decision architecture), the think approach (internalize new social norms), and the challenge of building new collective identities.

Keywords: non-compliant behavior; culture of lawfulness; nudge; think approach; social identity change theory

\section{Sumario}

\section{Introducción}

2. Aportes teóricos para entender las causas del incumplimiento
3. Herramientas políticas para una cultura de la legalidad: nudges, think \& identity change

4. Conclusiones

Referencias bibliográficas

\section{Introducción}

La cultura de la permisividad, de la complicidad, de la discrecionalidad, de la presión, del abuso y de la deshonestidad en el día a día de los ciudadanos, así como la negociación política de la ley, la politización de los procesos judiciales, la judicialización de la política, el clientelismo, la desobediencia de la ley, el afianzamiento de estructuras corporativas y la impunidad por parte de los que detentan el poder ejecutivo, legislativo y judicial, son, lamentablemente, comportamientos incumplidores constantes en gran parte de los países de América Latina, pero no solo en ella.

En un reciente informe del Banco Mundial, titulado Governance and the law, se insiste nuevamente en que el Estado de derecho y el imperio de la ley son pilares fundamentales que un buen gobierno necesita para desarrollar todo su potencial económico y social. Estudios empíricos, señala el informe, han revelado la importancia de que los gobiernos y los ciudadanos actúen de conformidad con la ley y de que existan condiciones en el ordenamiento jurídico que mejoren el funcionamiento de determinadas instituciones, que impulsen el crecimiento, que generen seguridad, que amplíen el acceso al crédito y, en suma, que mejoren la calidad de la justicia (Banco Mundial, 2017). Sin embargo, no es suficiente con dirigir los esfuerzos a la creación o al fortalecimiento de instituciones legales, también es necesario trabajar en preferencias, expectativas, creencias y cambios culturales.

Este marco general, sintéticamente descrito, constituye el trasfondo de este trabajo, cuyo contenido específico se concreta en dos objetivos. El primero 
de ellos es presentar y sistematizar enfoques analíticos que desde la investigación académica se han desarrollado para explicar el incumplimiento de las normas legales. En particular, interesa conocer, de acuerdo con académicos especializados, qué emociones y razones existen detrás de los comportamientos cumplidores e incumplidores de la legalidad. Una vez sistematizadas estas aportaciones, indicamos algunas de las propuestas políticas que cada enfoque propone para revertir el incumplimiento. Nuestro segundo objetivo consiste en conectar tres estrategias de gobernanza, consideradas soft, con el desafío de revertir el incumplimiento y promover una cultura de la legalidad.

La cultura de la legalidad puede ser entendida desde una lectura de máximos o desde una lectura de mínimos, y ambas se requieren y complementan. La de máximos dirige su mirada tanto a la construcción de un proyecto, en el «sentido de representar en perspectiva algo que se considera importante y que en consecuencia se pretende ejecutar en forma de principios y prácticas», como a la puesta en escena de un movimiento que podemos entender "como el desarrollo y propagación de una tendencia política, social y jurídica de carácter innovadora». En este proyecto y en este movimiento concurren, en diversos sentidos, múltiples fuerzas que adoptan la forma de instituciones, procesos, estructuras y valores y en cuyo vigor participan, por un lado, dimensiones de legitimidad y legitimación tanto jurídica como política; por el otro, condiciones de legalidad donde se entrecruzan escenarios relativos al entramado institucional, a la lucha contra las transgresiones de la ley y sus consecuencias sociales, y nuevas maneras de producción normativa y de apertura a mecanismos de pluralismo jurídico; $y$, finalmente, perspectivas en torno a la dinámica cultural de la cultura de la legalidad (Wences y Sauca, 2014: 17). Desde una lectura de mínimos, la cultura de la legalidad adquiere un valioso significado operativo que engloba los «conocimientos compartidos y los esquemas de comportamiento creados por los grupos sociales para percibir, interpretar, expresar y responder a las realidades sociales que les rodean» (Villoria y Jiménez, 2014: 88) y que tienden a generar conductas comunes, especialmente con relación a las expectativas, las creencias y los comportamientos tanto de los ciudadanos como de los gobernantes y de los órganos especializados en la ejecución del derecho con relación al respeto y al cumplimiento de la normatividad jurídica y de las prácticas políticas (Villoria e Izquierdo, 2015).

El fin último de este trabajo es contribuir a la construcción de una cultura de la legalidad en tanto proyecto y movimiento. Sin embargo, el análisis y las propuestas que se esbozan transitan sobre un eje operativo cercano a la lectura de mínimos. En la primera parte, examinamos contextos en los que se enraízan comportamientos incumplidores. En concreto, dirigimos la mirada a la ineficiencia del gobierno; la existencia de un orden o institucionalidad informal o paralela; el umbral de deshonestidad; y/o la falta de sincronía entre motivos (morales, éticos y legales) e incentivos. En la segunda parte, esbozamos propuestas de gobernanza soft que podrían ayudar a combatir estas tenencias incumplidoras. Nos detenemos concretamente en tres enfoques. Por un lado, las nudges (intervenciones en la arquitectura de decisión); por otro lado, el 
enfoque think (interiorizar nuevas normas sociales); y, finalmente, el desafío de construir nuevas identidades colectivas. Conviene advertir, finalmente, que los casos y ejemplos que acompañan este trabajo se centran en gran medida en experiencias latinoamericanas, aunque no solo en ellas.

El problema del incumplimiento normativo no es exclusivo de América Latina, pero en esta región es un problema de larga data que afecta gravemente a las democracias y obstaculiza las iniciativas de lucha contra la desigualdad, la inseguridad y la pobreza. Intentar solucionar esta compleja y dramática situación ha conducido, en los últimos años, a la emergencia y la consolidación de un corpus académico que congrega a historiadores, economistas, juristas, antropólogos, filósofos y otros científicos que han hecho aportaciones altamente significativas, no siempre conocidas o reconocidas más allá de sus fronteras. Las referencias teóricas de autores latinoamericanos ponen de manifiesto el interés de estudiosos de la región por entender el por qué somos como somos, qué nos ha llevado a esto y qué podría hacerse para revertir el comportamiento incumplidor. Ahora bien, las perspectivas que se delinean para explicar el incumplimiento y los mecanismos de solución al mismo nacen en escenarios diversos (incluyendo el mundo anglosajón) y resultan de interés y utilidad en muchos escenarios geográficos. Conectar este conocimiento es también meta de este trabajo.

\section{Aportes teóricos para entender las causas del incumplimiento}

En este apartado esbozamos cuatro perspectivas que nos ayudan a comprender los mecanismos explicativos del incumplimiento colectivo. Nos sirven de inspiración tres propuestas teóricas de académicos latinoamericanos que, desde distintos enfoques, ofrecen explicaciones sobre las razones que llevan al incumplimiento, así como los efectos y las consecuencias de dicho incumplimiento: la anomia boba de Carlos Nino, las prácticas contingentes que configuran la moralidad de Fernando Escalante y las mentalidades incumplidoras de Mauricio García Villegas.

La primera de las propuestas viene constituida por el conocido trabajo del argentino Carlos Nino (1992), quien dirige su atención a los costos colectivos que tiene la cultura del incumplimiento. Su idea de anomia boba hace referencia a una tendencia recurrente de sociedades latinoamericanas a la inobservancia de normas jurídicas, morales y sociales que de ser cumplidas conducirían a una acción colectiva más beneficiosa para el conjunto de la sociedad. Esa inobservancia de la norma, que se manifiesta en la economía informal, en la evasión impositiva, en corporativizar la economía, en la corrupción, en el incumplimiento de las reglas de tránsito vial, etcétera, produce disfuncionalidades en la sociedad y tiene una estrecha vinculación con el subdesarrollo. Los sujetos no cumplen en cuanto consideran que existe la probabilidad de incumplimiento por parte de los otros y, al mismo tiempo, las autoridades suelen convalidar este tipo de tendencias con moratorias impositivas, amnistías $\mathrm{o}$ indultos a diversas faltas, entre otras decisiones. 
Las propuestas que el autor plantea encarar para combatir la anomia son múltiples, sin embargo, quizás la más interesante y sugerente es la relacionada con una educación normativa que desde una perspectiva kantiana estimule a ciudadanos que estén dispuestos a hacer lo que creen correcto, aun cuando sea previsible que los demás no lo hagan, especialmente si se convierten en sujetos que pueden servir de referencia al resto. La anomia es profundamente antidemocrática y es preciso que los sujetos: 1) entiendan por qué las normas son necesarias para la cooperación, por qué la cooperación es necesaria para alcanzar resultados socialmente eficientes y por qué la democracia otorga legitimidad a las normas; 2) conozcan las normas en cuestión y la finalidad de las mismas, así como el proceso deliberativo que se encuentra en su origen; 3) detecten cuál es el comportamiento de lealtad normativa que equilibra la observancia de la conducta con la satisfacción de los fines normativos; 4) desarrollen la convicción sobre la adecuación moral de la observación normativa y la disposición a reprochar a quien no satisface la observancia (Nino, 1992). Solo la discusión pública como proceso inherente a la democracia puede romper el círculo autofrustrante de la anomia.

La segunda propuesta teórica que consideramos importante recuperar está representada por el trabajo del hispanomexicano Fernando Escalante, quien, en su conocida obra Ciudadanos imaginarios. Memorial de los afanes y desventuras de la virtud y apología del vicio triunfante en la República mexicana: tratado de moral pública (2005), publicada originalmente en 1992, reconstruye sociológicamente las raíces históricas del incumplimiento formal en México. En este trabajo, señala que la moralidad pública es una configuración histórica, resultado de una práctica habitual en la que operan acuerdos contingentes sobre cómo hacer las cosas y cómo comportarse, así como sobre lo que es bueno y lo que es malo. No son acuerdos que respondan a un diseño racional, sino consecuencias no intencionadas de la acción que pueden o no ser explícitas, que se producen y reproducen imperceptiblemente y que, en general, no pueden cambiarse ni variarse voluntariamente (Escalante, 2005). Lo relevante del trabajo de Escalante para los fines que ahora nos ocupan es su empeño en demostrar cómo, colateralmente al orden formal que determina el aparato jurídico, la vida se organiza informalmente por medio de relaciones y prácticas regulares con todas las características de un orden efectivo.

La tercera propuesta la constituye el interesante trabajo sobre la cultura del incumplimiento de Mauricio García Villegas (2010), quien analiza la mentalidad incumplidora y elabora creativas tipologías que sirven para clasificar a los incumplidores y ver cómo se conjuntan intereses, valores y resistencias políticas. Las tipologías han sido elaboradas con base en los probables razonamientos que hace el incumplidor de acuerdo con sus intereses, valores, creencias, así como por las circunstancias estructurales que le rodean. García Villegas sostiene que los motivos que llevan al incumplidor a realizar determinadas acciones y a comportarse de determinada manera se correlacionan con la clase social y con un determinado tipo de racionalidad, y señala, además, que las formas de actuar son el reflejo de deficiencias básicas del sistema político y jurídico. 
El autor colombiano sistematiza tres tipos ideales de incumplidores: el vivo, el rebelde y el arrogante. El vivo, incumplidor más frecuente en América Latina, es el aprovechado que busca satisfacer su propio interés y ante el cual la sociedad siente ambivalencia. Su razonamiento es el del calculador que sopesa el costo y el beneficio del incumplimiento de la norma, y su comportamiento denota la incapacidad institucional de los Estados para sancionar estos hábitos desviados. El segundo tipo es el rebelde y alude a aquel que no reconoce la autoridad de la norma, que se resiste ante el poder o que desconoce la legitimidad de las instituciones. El último tipo ideal es el arrogante. Los que responden a esta clasificación valoran la ley, pero consideran que son los otros los que deben cumplirla, ya que presumen que ellos, dados sus privilegios o condiciones, se encuentran por encima de esta.

En muchas sociedades latinoamericanas estos comportamientos no son la excepción, sino que están muy extendidos, al grado de haberse convertido en actitudes y hábitos tan interiorizados y frecuentes que los individuos, aun deseándolo, difícilmente pueden desprenderse de ellos.

En este artículo avanzamos sobre la huella que estos trabajos han dejado. Nos centramos en analizar el incumplimiento generalizado y ampliamente tolerado por la sociedad, la cual, a su vez, se acompaña de un deseo, también generalizado, de que el comportamiento social cambie hacia un modelo en el que todos cumplan la ley (Rothstein, 2000). Nos interesa particularmente tratar al incumplimiento, en el marco de un Estado social y democrático de derecho, como un hecho social que trasciende a los individuos y que debe entenderse y explicarse como fenómeno colectivo, aun cuando las explicaciones acudan también a la psicología de los sujetos.

Para comprender los mecanismos explicativos del incumplimiento colectivo, hemos delineado cuatro perspectivas. Para el diseño de nuestra clasificación tomamos en cuenta la concepción de la naturaleza humana y la manera en que el hombre considera que debe actuar para alcanzar sus fines, tanto individual como colectivamente. Conviene advertir, desde el principio, que estas lecturas nos son excluyentes, sino que se entrecruzan e incluso llegan a complementarse.

La primera perspectiva, a la que denominamos enforcement, dirige su atención al fallo de la organización gubernamental encargada de garantizar el cumplimiento de la norma y de penalizar a los que no cumplen. La segunda perspectiva se centra en la institucionalidad paralela que existe en muchas sociedades y que compite o anula la institucionalidad formal. Este enfoque, centrado en la informalidad paralela, tiene semejanzas con el incumplidor arrogante de García Villegas y comparte diagnóstico con la reconstrucción histórica que sobre el incumplimiento cultural lleva a cabo Escalante. La tercera perspectiva es el resultado de múltiples experimentos sociales que se fueron desarrollando para conocer la persistencia de determinadas conductas y comportamientos deshonestos, así como la psicología de los sujetos en la mediana de comportamiento social. Aquí el umbral de la deshonestidad se vincula con la identidad de los sujetos y varía en función de los controles legales y morales que regulan el comportamiento de los individuos. La última perspectiva, bau- 
tizada como desconexión, combina razón y emoción y se centra en el divorcio que existe entre los regímenes reguladores de la conducta como son la ley y lo socialcultural, por una parte, y la moral, por la otra. Veamos con más detalle cada una de estas perspectivas.

\subsection{Enforcement}

Esta perspectiva se centra en la percepción sobre la eficacia del gobierno. Cuando los sujetos consideran que existe un sistema legal y un proceso judicial que perciben como justos (buenas leyes, juicios imparciales, seguridad jurídica y procesos transparentes), pero, especialmente, un sistema eficaz (enforcement) que persiga, capture y castigue a quienes violan las normas, el incumplimiento es menos probable (Bergman y Rosenkrantz, 2009).

Lo que sostienen quienes se identifican con esta perspectiva es que cuando la probabilidad real de sanción y castigo es alta, y la impunidad y la ganancia que podría obtenerse de violar la norma es menor, hay una especie de racionalización del coste/beneficio que desanima el incumplimiento. Cuando lo que se gana incumpliendo es mucho o puede justificarse moralmente, el riesgo parece merecer la pena, y entonces la probabilidad de incumplir aumenta. Asimismo, cuando la probabilidad de ser capturado y sancionado es baja porque las instituciones son poco eficientes en sus labores o están diseñadas de una manera que resulta fácil escapar al castigo, las probabilidades de incumplimiento aumentan. En esta situación, no hay costos para quienes infringen las normas ni tampoco incentivos para cumplirlas (Bergman y Rosenkrantz, 2009).

Detrás de esta lectura prevalece una concepción del hombre como ser racional que evalúa alternativas de acción antes de comportarse de un modo u otro y toma sus decisiones con base en los costos y ganancias. En consecuencia, podríamos decir que las fallas o falta de capacidad de los organismos encargados de perseguir y castigar a los incumplidores y la consecuente desconfianza ciudadana en estas instituciones favorecen tanto el oportunismo como el escepticismo.

Esta perspectiva nos ayuda a comprender la cultura de la legalidad como un fenómeno asociado y dependiente de la eficacia de la ley. La aplicación deficitaria del derecho no solo no incentiva el acatamiento, sino que envía señales contrarias a la construcción de una cultura del cumplimiento.

Las soluciones que se proponen en el marco de esta perspectiva son extensamente conocidas y, desde hace tiempo, se vienen formulando en marcos de lucha contra la corrupción: diseño de instituciones que eviten discrecionalidad; dotar de mayores recursos financieros y humanos tanto a las instituciones encargadas de perseguir a quienes violan la ley como a aquellos que se encargan de castigarlos (policía y poder judicial); formación y capacitación de estos dos cuerpos para mejorar la eficiencia e imparcialidad; mayores controles internos y externos de estas instituciones; refuerzo de formación en ética pública; desarrollo de instituciones específicas para atender ciertos delitos que afectan bienes y valores públicos como la corrupción (contralorías y fiscalías), etcétera (Villoria e Izquierdo, 2015). 
Sirva de ejemplo el caso mexicano, donde en los últimos años se han creado nuevas organizaciones estatales con recursos financieros y humanos destinados a luchar contra la corrupción y a favor de la transparencia, como son el Instituto Nacional de Transparencia, Acceso a la Información y Protección de Datos Personales (nacido en 2003 con otro nombre) y el Sistema Nacional Anticorrupción (puesto en marcha en julio de 2016).

\subsection{Informalidad paralela}

El estudio de instituciones informales, descritas por Douglass North (1993) como aquellas reglas que gobiernan los comportamientos por fuera de los canales oficiales, ha obtenido bastante interés por parte de la literatura académica desde hace unos años y coincide en buena medida con las ideas de Fernando Escalante antes expresadas. North, uno de los fundadores del nuevo institucionalismo, comprende con bastante claridad la importancia de la cultura en la acción individual y en la acción colectiva y la percibe como un componente central para entender el cambio social tanto a corto como a largo plazo. De acuerdo con esta lógica, las instituciones (formales e informales) son límites y guías para la conducta humana en sociedad. Conocidas y evidentes para los actores sociales, al igual que las reglas formales, las instituciones informales descansan en un sistema de sanciones y beneficios que provee incentivos a los sujetos para que se comporten de determinadas maneras.

En esta misma línea argumental se manifiesta Guillermo O'Donnell, quien llama la atención sobre el importante hecho de que, en las democracias latinoamericanas, el particularismo coexiste en incómoda tensión, pero perfecto equilibrio, con y dentro de las instituciones y reglas formales (O'Donnell, 1999). En esta coexistencia, intervienen rituales y discursos en los que las reglas informales pasan a ser la principal guía de comportamiento y crecen actitudes y prácticas que tienden a propiciar un alejamiento, cuando no una plena contradicción, entre las reglas no escritas establecidas por la costumbre y convertidas en parte esencial del sistema y las normas legales.

Este entramado de instituciones informales que coexisten y subsisten pese a los intentos por impulsar a otras lógicas donde prevalezca el imperio de la ley, y que deja en los individuos una permanente sensación de impotencia y frustración, tiene raíces históricas que han dejado como herencia, entre otras cosas, una personalización de la autoridad, una organización jerárquica de la vida social, una defensa tradicionalista del orden local, una lógica clientelar de las relaciones políticas y un recelo permanente frente a la autoridad formal (Escalante, 2005).

Este sistema de usos, costumbres y formas de acción que regula los comportamientos sociales y les dota de sentido va moldeando una moralidad pública que da cauce a una vida colectiva que ayuda a sortear los problemas de autoridad, jerarquía y coexistencia en numerosos países latinoamericanos. Sus enraizadas estructuras chocan frontalmente con los intentos por construir una cultura de la legalidad. 
Ajustado a la teoría de la acción colectiva, este enfoque se centra en lo que se denomina problemas de segundo orden, donde todos los actores entienden que estarían mejor cumpliendo la ley, pero, como no pueden confiar en que los otros lo harán, entonces no ven razones para cumplir. Si la moralidad pública es un constructo histórico, es muy difícil cambiar rutinas, compromisos tácitos y emociones. Esta trampa política y el poder inercial de los path dependence nos recuerdan que el cambio en estos escenarios es complejo y depende del aprovechamiento de una coyuntura crítica que permita poner en marcha una reforma con capacidad para cambiar las creencias más profundas sobre «qué esperar de los demás» y construir una expectativa favorable sobre las acciones de los otros. Para alcanzar este objetivo, especialistas hablan, por un lado, de crear en las sociedades un «nuevo juego» que combine mecanismos formales e informales de monitoreo y sanción; $y$, por el otro, de crear sistemas de reciprocidad y confianza (Persson et al., 2013).

El artículo de Persson y su equipo cita experiencias en Suiza, Dinamarca, Estados Unidos, y recientemente Hong Kong y Singapur, como ejemplos de transición exitosa de sistemas corruptos a sistemas ejemplares. En todos ellos se hicieron amplias reformas políticas, económicas y sociales que incluyen sistemas formales e informales de control y sanción, así como de confianza y reciprocidad. En los casos de cambio exitoso, los actores que se ubican en la parte superior del sistema, miembros de la elite gobernante y funcionarios públicos de alto nivel, han servido como modelos a seguir al cambiar su modelo de comportamiento. A partir de allí, los ciudadanos se dieron cuenta de que había un «nuevo juego» en la ciudad y el modus operandi de las instituciones pasaba del particularismo y la arbitrariedad al universalismo y la imparcialidad (Persson et al., 2013: 465-467). La clave en este enfoque es el cambio radical de reglas y el comportamiento de quienes deben dar el ejemplo.

\subsection{El umbral de honestidad}

Experimentos llevados a cabo por académicos como Dan Ariely demuestran que, lejos de existir «manzanas podridas» que contaminan con intensidad la media de comportamientos honestos, lo que hay es una mayoría de personas que hace trampa, pero solo un poquito (Ariel, 2014).

Las personas son honestas solo en la medida en que les conviene y no basan tal decisión en un análisis de coste-beneficio. Nuestro monitor de honestidad interno únicamente se activa cuando contemplamos la posibilidad de realizar grandes transgresiones, pero no cuando llevamos a cabo pequeños comportamientos deshonestos. De acuerdo con Ariely, deseamos cometer actos deshonestos sin sentirnos deshonestos; cometemos pequeñas faltas y actuamos deshonestamente siempre que no alteren la opinión que tenemos de nosotros mismos. Cuando percibimos que el resto también lo hace, sentimos legitimado nuestro comportamiento. El engaño no solo es común, sino también infeccioso y puede aumentar y expandirse cuando observamos la mala conducta de quienes nos rodean. Esta actitud, conocida como reflejo condicionado, muestra 
que la conducta emocional se encuentra generalmente condicionada por el entorno social. Las fuerzas sociales circundantes funcionan de dos maneras: si el tramposo es integrante de nuestro grupo social, nos identificamos con él y engañar nos parece más aceptable (especialmente si esa persona tiene una posición de autoridad). Si es un intruso, cuesta más justificar la conducta y cambiamos nuestro comportamiento movidos por el deseo de distanciarnos de esas personas que consideramos inmorales (especialmente si son vistos como rivales). Esto pone de manifiesto lo decisivas que son las otras personas en nuestra actitud (Ariely, 2012).

En resumen, esta lectura de la deshonestidad, que busca explicar la conducta de los individuos en ciertos escenarios de interacción — correlacionando autoestima, identidad y valoración personal con oportunidades contextuales-, conduce a explicar las diferencias entre niveles de cultura de la legalidad. No tanto con relación a los individuos que componen las sociedades, sino con relación a la permisividad o falta de control que esas sociedades tienen y a su capacidad para incentivar códigos morales guiados por conductas honestas. Lo que mejor funciona para evitar este tipo de comportamientos son, de acuerdo a sus experimentos, los recordatorios morales y la vigilancia y el castigo inmediato, incluso ante pequeñas faltas.

En el marco de esta perspectiva, las acciones recomendadas para reducir la deshonestidad, poner a cero nuestra brújula moral y superar el efecto "qué demonios» ${ }^{1}$ se centran en los resultados obtenidos en experimentos sociales. En ellos se destacan cuatro vías que evitan los deslices hacia la tentación de hacer trampa: a) firmar una declaración antes de desarrollar una tarea o comenzar un trabajo, en donde el sujeto se compromete a compotarse con honestidad; b) recordatorios de reglas morales como los diez mandamientos o juramentos éticos profesionales, que recuerden a los individuos su deber social con el colectivo al que pertenecen; c) supervisión y control externo de los actos; d) regular los conflictos de intereses que debilitan los compromisos morales teniendo en cuenta el efecto que el entorno y el agotamiento físico y mental tienen sobre los sujetos. Para esta perspectiva, las reformas que intentan cambiar abruptamente nuestro comportamiento son ineficaces porque no entienden la psicología de los individuos que, frente a la nueva norma, buscarán la manera de eludirla (Ariely, 2014; Ariely, 2012; Mazar et al., 2008).

Un ejemplo ilustrativo de cómo operan algunas de las recomendaciones sugeridas lo ofrece el propio Ariely a partir de un experimento desarrollado en Sudáfrica para potenciar una alimentación saludable donde se desplegó un programa voluntario de compromiso previo. Se ofertó a los miembros de Discovery Vitality un descuento del 25\% en comestibles preseleccionados si aceptaban aumentar sus compras de alimentos saludables en 5 puntos porcentuales por encima de la línea base de su hogar durante 6 meses. Los miembros que no

1. El efecto "qué demonios» sugiere que una vez empezamos a infringir (por ejemplo, no pagando el transporte público o tirando las pilas en los contenedores inadecuados) se allana el camino para seguir e incrementar nuestra conducta incumplidora (Ariely, 2012: 131). 
se inscribieron en este programa de compromiso previo recibirían igualmente su descuento. El objetivo era ver cómo se comportaban los que se inscribieron agregando un obstáculo voluntario a sus selecciones futuras. Los resultados indicaron que el 36\% de los miembros decidió participar, y con relación a los que no participaron estos mantuvieron un aumento de 3,5\%, en promedio, en artículos de comestibles saludables comprados en cada uno de los 6 meses. Estos hallazgos sugieren que una parte de los consumidores aprovechó la oportunidad de crear ambientes de elección restrictiva para sí mismos (Schwartz et al., 2014). En el caso de los recordatorios de códigos morales y éticos, estos pueden ser de utilidad para garantizar comportamientos más honestos en ciertas profesiones, no solo con los médicos y académicos, sino también en el mundo de las finanzas o de las empresas público-privadas. Lo importante para el caso es que el recordatorio sea recurrente (reiterado en el tiempo) y anterior al desarrollo de una acción, de modo que permita una mayor supervisión del propio comportamiento. En el caso de los controles y vigilancias, este tipo de recomendaciones vale para casi todo tipo de comportamiento. Desde normativas viales donde la presencia de un vigilante o cámara de seguridad sirve para garantizar que la gente use el cinturón de seguridad o respete la velocidad y los pasos peatonales, a comportamientos donde el control es más difícil por ser menos visible, como los vinculados a la piratería informática (descarga de música o documentos con propiedad intelectual).

Los citados trabajos del equipo de Ariely son enfáticos en demostrar que las pequeñas mentiras pueden convertirse en enormes bolas de nieve cuyos alcances pueden ser cada vez más difíciles de revertir y, por ello, estiman que lo más aconsejable es afrontarlas cuando son pequeñas. Los resultados y aprendizajes obtenidos a lo largo de sus experimentos comparados no se circunscriben a determinadas culturas, sino que han demostrado ser válidos para sociedades muy diferentes ${ }^{2}$. Ahora bien, las propuestas que ofrecen para trabajar sobre la irracionalidad, el engaño y el incumplimiento parecen ganar mayor interés en sociedades donde el Estado de derecho y la cultura de la legalidad están más consolidados, y no tanto en sociedades donde los Estados se definen como fallidos, los gobiernos son ineficaces y la impunidad y la corrupción están extendidas. En todo caso, creemos que incluso en estas sociedades las recomendaciones ofrecidas pueden coadyuvar a mejorar la honestidad y el cumplimiento de normas en ámbitos como los mencionados.

\subsection{Desconexión}

En este enfoque, la regulación legal es explícita, escrita, crea derechos y deberes públicos; tiene vigencia definida y castigos conocidos para casos de incumplimiento; la emite y la aplica una autoridad expresamente facultada para ello; y rige formalmente nuestras sociedades. La regulación moral, por su parte, es

2. Para más información, ver http://thedishonestyproject.com/y http://danariely.com/resources/apps/ [Consulta, octubre de 2018]. 
implícita o reconstruida a partir de la reflexión; su incumplimiento se penaliza desde la propia conciencia generándose culpa, vergüenza, malestar interno, exclusión y tensión en la identidad personal. La forma de invocar esa regulación es mediante las emociones y los deberes morales.

La nota característica de esta perspectiva sugiere que los sujetos actúan racionalmente; saben que cumplir la ley es socialmente beneficioso, aun cuando en su foro interno parecen preferir no cumplirla porque suponen o imaginan que los demás no la cumplirán.

Para esta perspectiva, John Elster nos sirve de primera orientación. La influencia de la cultura es incorporada por este autor a su concepto de racionalidad, construido como resultado de su crítica a la economía clásica y a la propia teoría de la elección racional de la que forma parte; para él, la acción racional es instrumental, es decir, está orientada a los resultados, pero también está guiada por normas sociales. Cuando la gente obedece normas, subraya, «a menudo tiene un resultado particular en la mente: desean evitar la desaprobación de la gente. La conducta guiada por la norma es apoyada por la amenaza de las sanciones sociales que hacen que sea racional obedecer las normas» (Elster, 1990: 120). En esta lógica, compartida por otros defensores del nuevo institucionalismo, lo que explica la acción son los deseos de la persona junto con sus creencias acerca de las oportunidades.

Esta cuarta perspectiva es quizá la que mejor integra los aspectos racionales, emocionales y sociales que subyacen tras la conducta humana a la hora de explicar por qué falla la acción colectiva y escasea la cooperación (y por qué no se cumplen las normas que, de cumplirse, beneficiarían al colectivo). De acuerdo con John Elster, para el grupo es mejor si todos cooperan, aun cuando no cooperar puede generar más beneficios individuales y la posibilidad de que se presenten conductas oportunistas en la acción colectiva.

Tomando como referencia estas ideas y con un sentido eminentemente práctico, Antanas Mockus, exalcalde de Bogotá y recién elegido senador, formulará su teoría del divorcio entre órdenes regulativos: legal, moral y cultural para explicar el incumplimiento (Mockus, 1999). La regulación legal emana de una autoridad facultada y de un proceso previsto, protege derechos y repara mediante sanciones; la moral se sustenta en la propia conciencia e invoca emociones y repara a través del arrepentimiento; la cultura se enraíza en la comunidad de pertenencia e invoca hábitos y creencias compartidas y repara mediante disculpas o ritos de expiación. Sincronizar el orden legal con el moral y el cultural es la clave para incrementar y mejorar el cumplimiento ciudadano.

El reto para Mockus es construir puentes que los hagan compatibles; sincronizar estos distintos sistemas que nos regulan, mediante la construcción de una nueva sociabilidad, es fundamental para crear cultura de la legalidad. Este reto solamente podrá alcanzarse fomentando nuevas representaciones (formas de decir, proyectar y evaluar la realidad), modificando actitudes, comportamientos y hábitos, y erigiendo una visión compartida que ayude a otorgar sentido y orientación al cambio cultural. Mockus sostiene que para generar cultura de la legalidad hay que enfatizar tanto el aspecto positivo como 
el negativo de la motivación. Deben promocionarse conductas que susciten un apego por la ley, una sensación de gratificación por la buena acción y un reconocimiento social. En cambio, el aspecto negativo de la motivación consiste en incentivar el temor a la sanción legal y a la censura social, así como en despertar la culpa por la mala conducta. Dar solidez a la cultura de la legalidad, y revertir su debilidad, requiere incentivos legales, pero también morales, racionales y emocionales.

En el programa de cultura ciudadana implementado durante su gestión en Bogotá, se propuso una intensa campaña de comunicación sobre los objetivos de la ley y sobre las formas de aplicación de la ley con la finalidad de aumentar el cumplimiento voluntario de normas, el cumplimiento de acuerdos pacíficos concertados entre ciudadanos y la ayuda mutua. Este programa buscaba, por un lado, influir en la conducta de los ciudadanos, especialmente promoviendo la autorregulación a fin de fomentar compromisos morales; $y$, por el otro, impulsar una batería de acciones que visibilizaran a los altruistas y a los cooperadores (Mockus, 1999). También fue importante el impulso, mediante la regulación cultural, de lo que se consideraban acciones correctas. Ejemplos de este tipo de políticas destinadas a suscitar compromisos morales autogratificantes fueron la cultura tributaria voluntaria, el ahorro de agua o el desarme voluntario. $\mathrm{Y}$ ejemplos de acciones destinadas a instaurar normas colectivas de carácter cultural son las tarjetas y actuaciones de mimos para el control del tránsito (Mockus, 2006). Esto último forma parte de una serie de aliados que se consideraron indispensables en esta campaña: el arte, el humor y lo lúdico (Mockus, 2003).

Los «caballeros de la cebra» y las actuaciones de mimos son iniciativas ilustrativas de cómo las instituciones políticas buscan cambiar conductas haciendo confluir la ley con la moral y la cultura; en este objetivo se acude al reconocimiento social para recompensar comportamientos. Respecto al primer ejemplo, entre 1995-1997 se llevó a cabo la campaña en contra de los crímenes relacionados con taxistas. Esta campaña permitía identificar y premiar a los taxistas que cumplieran con las normas y que ofrecieran un servicio encomiable a la comunidad. Para ello, la Administración reunió a 150 taxistas ejemplares y los nombró «caballeros de la cebra». Se les encomendó la misión de buscar otros taxistas que se comportaran como ellos y convencer a quienes no lo hacían. El segundo caso consistió en usar tarjetas y acudir a artistas que mediante actuaciones de mímica participaron en el control del tránsito; esta iniciativa buscaba visibilizar conductas mediante la conversión de la norma legal en norma social. Cuando un vehículo se detenía en la senda peatonal en infracción, el mimo hacía la mímica de empujar el auto hacia atrás, para que dejara de pisar la senda peatonal. También se utilizaban unas tarjetas rojas grandes, si su accionar era inapropiado, o blancas, si su conducta merecía la aprobación de toda la ciudadanía. En general, los peatones se sumaban al mimo y apoyaban su acción. Si el conductor se mostraba intransigente, había agentes de tránsito escondidos que salían y ponían la multa, y la gente, por primera vez, aplaudía a un oficial que multaba a alguien. 
Hasta aquí hemos agrupado aportes que desde distintos ángulos disciplinares y experiencias políticas se han diseñado para explicar las razones de la cultura del incumplimiento y hemos esquematizado sus potenciales respuestas políticas. En términos generales, las tres primeras perspectivas apelan a herramientas políticas clásicas como el desarrollo de castigos o incentivos para reconducir la conducta humana hacia objetivos sociales. El modo tradicional de regular la vida social son las leyes, y en ellas la herramienta clásica que se utiliza para guiar y moldear los comportamientos individuales son las prohibiciones, mandatos o incentivos económicos (positivos y negativos). Estas últimas descansan en el deseo de los sujetos de evitar el castigo o pérdida que supone el incumplimiento. El incumplimiento normativo, del que venimos hablando, da cuenta de la insuficiencia de estas herramientas para alcanzar sus metas. Por esta razón, incorporar elementos que tengan en cuenta aspectos que van más allá de la racionalidad y el temor al castigo físico, social o pecuniario se vuelve interesante y prometedor.

En el apartado que sigue analizaremos una serie de herramientas políticas que gravitan en torno a la heurística, a los límites cognitivos, a la reflexión individual sobre la conducta, y al debate grupal sobre lo adecuado y deseable en una sociedad. Dichas herramientas no responden a una perspectiva específica de las cuatro antes comentadas y, por tanto, pueden pensarse útiles para cualquiera de ellas. La pertinencia y la oportunidad de estas herramientas dependerán del contexto social en el que hay que resolver el problema, y corresponderá a los diseñadores de políticas públicas la selección de una u otra herramienta o la combinación de ellas. En suma, este catálogo de nuevas herramientas que presentamos puede ser útil para combatir el incumplimiento de la ley y sumarse a los tradicionales esfuerzos centrados en castigos, prohibiciones e incentivos.

\section{Herramientas políticas para una cultura de la legalidad: nudges, think \& identity change}

Hay tres tipos de herramientas políticas catalogadas como soft que pueden ayudar a revertir el círculo vicioso de los comportamientos incumplidores y transformar expectativas y creencias. En primer lugar, la nudge, estrella de los últimos tiempos, deudora de la economía conductual y de la psicología social y enraizada en la filosofía del paternalismo liberal. En segundo lugar, las políticas think, enfoque procedente de la teoría política normativa asociada a la democracia deliberativa que confía en que los ciudadanos, si tienen la información suficiente, el tiempo necesario y el contexto más apropiado, pueden llegar a los mejores juicios acerca de lo que es bueno para ellos y sus conciudadanos. Por último, desde una perspectiva analíticamente diferente, nos referiremos a quienes proponen un cambio en la identidad social a partir de la reflexión autoconsciente como mecanismo alternativo de cambio social y que permite extraer pistas útiles sobre la creación de una cultura de la legalidad. 


\subsection{Nudge}

La palabra nudge suele traducirse al español como pequeño empujón o empujoncito y alude a las iniciativas que, sin coacción, guían las decisiones individuales hacia lo que se considera la correcta dirección, de acuerdo a como los mismos individuos lo juzgan, y siempre preservando la libertad de elección y sin hacer daño a otros (Thaler y Sunstein, 2009; Sunstein, 2016; Sunstein, 2017).

Los empujoncitos consisten en divulgar información, hacer advertencias y diseñar reglas que señalan qué sucede si las personas no hacen absolutamente nada; no obligan, más bien están dirigidos a razonar, persuadir e incluso disentir (Güemes, 2017). Las nudges se enmarcan filosóficamente en el paternalismo suave o libertario y en el paternalismo de medios, ya que preservan la libertad de elección ${ }^{3}$. Su objetivo es incrementar el bienestar individual (ayudar a que las personas tengan una vida mejor, alargar su salud, mejorar su calidad de vida) y social ${ }^{4}$, sin interferir en la libertad de elección. Cuando los arquitectos de la elección, esto es, las acciones del gobierno, intentan incrementar el bienestar imponiendo grandes costes sobre los que eligen, entonces estamos ante un paternalismo duro (por ejemplo, sanciones penales); en cambio, cuando las acciones del gobierno intentan aumentar el bienestar de las personas influyendo en sus elecciones pero sin imponer costes materiales o mediante costes muy pequeños (por ejemplo, cobrando unos céntimos por las bolsas de plástico en los supermercados o poniendo etiquetas de ahorro de combustible en los electrodomésticos), estamos ante un paternalismo suave que incluye empujoncitos.

Cuando el gobierno promueve nudges está intentando: a) influir en los resultados sin afectar a las acciones o a las creencias de las personas; b) influir en las acciones de las personas, sin que ello afecte a sus creencias; c) alterar creencias para influir en sus acciones; y d) modificar preferencias, independientemente de afectar a sus creencias, con el objeto de influir en sus acciones.

Ejemplo de lo primero son las reglas por defecto (default option) como un plan de ahorro o seguro de salud de afiliación automática de los trabajadores. Asumiendo la inercia con la que actuamos, el optimismo del presente y la subestimación del futuro, esta herramienta prevé un resultado que pretende ser beneficioso para el bienestar del destinatario. Una multa de tráfico por no usar el cinturón de seguridad o el casco cuando se conduce en moto entra en la segunda categoría porque afecta a lo que la gente hace, pero no necesaria-

3. «La arquitectura de la elección», subraya Sunstein, está presente en nuestras acciones vitales (cuando entramos en un restaurante, cuando escogemos nuestro ocio, cuando elegimos una hipoteca, cuando visitamos nuestras páginas web favoritas, etcétera) e influye de manera considerable en los resultados. La arquitectura de la elección es decisiva porque puede hacer que elijamos ciertas opciones y que esto pueda tener numerosos efectos sobre otras opciones al empujarnos, de manera consciente o no, en una u otra dirección. La arquitectura de la elección es inevitable, no se puede eliminar.

4. Con relación a qué se considera bienestar, Sunstein defiende una idea extensiva que se aleja de la idea estrecha de utilidad e incluye elementos de la vida que no necesariamente deben calificarse como de felicidad. Ahora bien, hay que diferenciar entre «el bienestar desde el punto de vista de quién elige y el bienestar desde el punto de vista del paternalista». 
mente afecta a lo que cree. Muestra de nudge que busca alterar creencias para influir en las acciones son los carteles que se encuentran en los baños de los hoteles detallando los efectos negativos que tiene lavar todos los días las toallas y animando a no dejarlas en el suelo. Por último, un tipo de nudge que busca modificar preferencias pero sin afectar sus creencias es una campaña gráfica que, mediante la alerta sobre el peligro de conducir fumando o hablando con el teléfono móvil, busca influir en las acciones con el objeto de que las personas prefieran no hacer estas cosas.

Con el fin de ilustrar esta herramienta, recogemos a continuación ejemplos concretos de nudges llevadas a cabo en América Latina.

En una investigación publicada por el Banco Interamericano de Desarrollo (Castro y Scartascini, 2014), se subraya que las nudges y lo que parecen pequeños detalles pueden ser estrategias interesantes para mejorar la adquisición de objetivos sociales que, a nuestro entender, se vinculan con la cultura de la legalidad. En este sentido, los autores destacan los resultados de experimentos de política conductual en tres municipios de la provincia de Buenos Aires donde se buscó mejorar la recaudación fiscal. Para ello, se planificó la introducción de mensajes en las facturas de servicios municipales con imágenes alusivas. Las evaluaciones posteriores demostraron que mensajes efectivos, sencillos, claros y acompañados por una imagen consonante mejoraban la recaudación. En el municipio de Junín, el mensaje más eficaz fue uno que tenía como objetivo disuadir del comportamiento mediante multas y potenciales medidas administrativas y judiciales que la municipalidad podría adoptar en caso de incumplimiento. El resultado fue que el cumplimiento tributario entre los contribuyentes que recibieron este mensaje disuasivo aumentó en casi 5 puntos porcentuales con respecto al grupo de control (Castro y Scartascini, 2014: 10).

En Colombia, Barrera-Ossorio y su equipo de trabajo (2011) realizaron pequeñas modificaciones a las políticas de transferencia social condicionadas a logros educativos. Tomando en consideración las dificultades de las familias receptoras, introdujeron dos variantes al diseño clásico. La primera ponía la mirada en los problemas de ahorro que tienen las familias y la consecuente inversión en material educativo. Para revertir esta tendencia, se retuvo un tercio del importe de los fondos totales a distribuir en el año y se les entregó a las familias en el momento en que debían hacer la inscripción en la escuela. La segunda variante consistió en retener el mismo importe, pero prometiendo a los destinatarios entregárselo pasada la graduación y una vez que estuvieran matriculados en una institución de educación superior. En ambos casos, la estrategia resultó exitosa. En el primero se incrementó en 9,4 por ciento la matrícula y en el segundo un 48,9 por ciento.

En Perú y Bolivia, estudios experimentales demostraron que el uso de mensajes recordatorios por móvil (SMS) aumentó las tasas de ahorro de las personas y permitió superar límites cognitivos como la inercia, la aversión a la pérdida o la subestimación del futuro. Los mensajes que destacaban objetivos específicos que los propios actores se habían fijado (por ejemplo, ahorrar para comprarse un televisor) fueron el doble de efectivos que los mensajes generales (Banco Mundial, 2015). 


\subsection{Think}

La estrategia de reflexión o pensamiento (think) sugiere que los gobiernos deben crear marcos institucionales en donde los individuos puedan visualizar sus limitaciones racionales y acceder a los puntos de vista de otros sujetos mediante la deliberación (John et al., 2011).

Quienes proponen esta iniciativa lo hacen en el marco de las críticas que la nudge ha recibido y que ellos mismos formulan. Para construir dicha perspectiva, los defensores del enfoque think recuperan los principios de la democracia deliberativa; de acuerdo con ellos, las preferencias no son fijas y exógenas, sino maleables y los sujetos se consideran razonables y capaces de pensar colectivamente y no seres con carencias y sesgos cognitivos de los cuales las normas deberían aprovecharse (John et al., 2009). La deliberación, constituida como fundamento de legitimidad de la decisión, dirige su mirada a las competencias desplegadas por los participantes en la discusión y su aptitud para formular argumentos racionales susceptibles de convencer a los otros. Supone, en la práctica, que se respeten diferentes condiciones con el fin de que la fuerza del mejor argumento pueda prevalecer en el intercambio. Entre estas condiciones, la democracia deliberativa reclama participantes activos, dispuestos al entendimiento y abiertos a los argumentos de otros, así como un ejercicio deliberativo de carácter público en el que todas las personas susceptibles de ser concernidas por las decisiones que se adopten deben tener las mismas oportunidades de acceder y de participar (Cohen, 1989).

De acuerdo con Peter John, la deliberación tiene efectos pedagógicos y educativos en los ciudadanos, ya que la necesidad de justificarse ante otros moldea sus perspectivas y preferencias egoístas, especialmente ante el público, y los conduce a pensar en términos más colectivos. Lo importante en estos casos es que el espacio de deliberación esté diseñado de manera tal que, por un lado, sea capaz de involucrar a los ciudadanos de diferentes grupos sociales (incluyendo grupos marginales) y, por el otro, disponga las condiciones que hagan posible el debate afable y abierto a opiniones contrapuestas.

La persuasión sería la herramienta blanda de la gobernanza que permitiría influir en los comportamientos y transformar hábitos compartidos. La persuasión es un arte que implica capacidad de raciocinio de los sujetos, habilidad para comprender nueva información y flexibilidad para alterar preferencias y creencias. Los ejemplos más comunes son el presupuesto participativo y la creación de minipúblicos como los jurados ciudadanos o las asambleas ciudadanas, que aseguran la inclusión buscando hacer partícipe a una muestra aleatoria de ciudadanos (John et al., 2011).

La deliberación permite conocer valores distintos a los propios y visualizar diferentes intereses, lo que puede ayudar a construir sentidos colectivos e impulsar comportamientos cívicos, aun cuando — sin duda - el costo de crear escenarios deliberativos es más alto que el de impulsar nudges, y requiere de condiciones exigentes como crear ambientes cómodos para quienes participan y hacer que todos los ciudadanos puedan participar y tener la misma capacidad de influencia, entre otros. 
Para ilustrar el enfoque, recogemos varios ejemplos. El citado trabajo Nudge nudge, think think. Experimenting with the ways to change civic behavior ofrece experimentos en Reino Unido en áreas vinculadas al voluntariado, donación de libros, estimulación del voto y el reciclaje, en donde el involucramiento ciudadano es altamente positivo (en zonas ricas y pobres y con diferentes tipos de personas). En el caso del reciclaje, se buscó estimular la colocación de residuos urbanos en los correspondientes contenedores. Para ello, se realizaron pruebas comparando nudges que usaban postales con caritas felices y tristes (se entregaban como respuesta al comportamiento del ciudadano) en una campaña educativa donde un grupo de personas iba puerta por puerta explicando la importancia de reciclar, suministrando información sobre los días de recogida de residuos, regalando bolsas para residuos y estimulando actitudes pro reciclaje. El experimento demostró que la lealtad y la búsqueda de identificación con el grupo - los vecinos- jugaron un papel importante (John et al., 2011: 147).

En referencia al ámbito iberoamericano, Brugué y sus compañeros describen una experiencia deliberativa a partir de la cual se diseñó un plan de gestión de las cuencas hídricas internas de Cataluña (Brugué et al., 2018). A fin de responder a la normativa europea, la Agencia Catalana del Agua constituyó una unidad específica para tratar el problema; esta tenía por finalidad informar y escuchar proactivamente la opinión de ciudadanos diversos, así como de los representantes de entidades, administraciones y empresas. Hubo cinco fases de trabajo: preparatoria, informativa, deliberativa, de conclusiones y documentación y de retorno. Amparados por unas reglas claras y por una adecuada gestión de las expectativas, se propició una situación donde había consenso sobre cómo abordar el desacuerdo y generar unas relaciones basadas en el respeto mutuo. El acuerdo sustantivo entre distintas posiciones no se logró, pero sí se propició una situación donde se aceptaron las diferencias y gestionaron la emergencia de nuevas actitudes.

Por último, conviene citar la literatura y estudios sobre los numerosos casos de presupuestos participativos (Sousa Santos, 1998; Goldfrank, 2006), asambleas ciudadanas (Hadad et al., 2012) y estrategias de $e$-democracy (Altman, 2002) presentes con fuerza en América Latina en las últimas décadas. La base de datos LATINNO (2017) recoge algunas de estas experiencias de innovación democrática donde, entre otros, destacan: los Diálogos Nacionales en Bolivia (una serie de mesas redondas organizadas por el gobierno para buscar un consenso en torno a la estrategia a largo plazo de la lucha contra el hambre y el desarrollo económico del país, y en la que participan autoridades, empresarios, organizaciones de la sociedad civil y comunidades indígenas a nivel municipal, departamental y nacional); las Mesas de Concertación y Lucha contra la Pobreza en Perú (foros de diálogo y negociación entre gobierno, representantes de la sociedad civil y ciudadanos, a través de los cuales se diseñan políticas sociales y programas económicos); y, por último, los Consejos de Desarrollo Rural Sustentable en México (instancias locales de participación en las que productores y agentes sociales del mundo rural toman decisiones sobre prioridades, planeamiento y distribución de recursos públicos asignados al desarrollo rural sustentable). 


\subsection{Identity change - steer}

La teoría de la identidad dirige su atención a la necesidad de interiorizar nuevos comportamientos ante las reglas y a generar una autoconciencia y una reflexión proactiva que modifique el comportamiento en el mediano y largo plazo (Mols et al., 2015).

La tercera de las herramientas que presentamos se nutre de la psicología social y se centra en la relación entre autodefinición de los sujetos, por un lado, y las normas, actitudes y comportamientos, por el otro. El comportamiento individual es guiado por la pertenencia a un grupo social y a sus normas, pero no en términos de pertenencia al grupo, sino de creencias normativas asociadas a la propia identidad.

De modo que, si lo social es fundamental para explicar el sentido y la dirección de los comportamientos individuales, la clave del cambio de comportamiento pasa por encontrar un terreno de identidad común y definir (o redefinir) ese terreno en una senda donde las nuevas normas se vuelvan aceptables como parte integral de un autoentendimiento compartido. Mols y los otros autores que escriben con él señalan que la identificación social hace posible la influencia social, pero más interesante que la adaptación (que solo funciona cuando la mayoría cumple las normas y solo existe un grupo pequeño de desviados) es la interiorización o aceptación personal de las normas.

La educación por pares es un buen ejemplo de cómo los diseñadores de políticas pueden trabajar con las normas existentes. Desde esta perspectiva, se considera que es más conveniente formar líderes de opinión o dar herramientas a los existentes, a fin de que influyan en sus pares, en lugar de imponer criterios desde arriba. En áreas como la educación sexual, el consumo de drogas o las actitudes racistas, este enfoque puede resultar muy útil en cuanto los actores de referencia tienden a persuadir al resto de que adopte nuevas normas y comportamientos como parte integral del «quienes somos nosotros», «lo que representamos» $y$, en consecuencia, «lo que hacemos» (Mols et al., 2015: 92).

En todos los casos, crear nuevas mentalidades comunes requiere: a) identificar los rasgos de una identidad social; b) mapear las normas existentes; c) identificar lo que se percibe como legítimo y lo que no; y d) determinar quiénes son los líderes de opinión en esa comunidad. Todo esto supone recursos y más dilación en el tiempo, aunque augura un mayor afianzamiento y una mayor perdurabilidad.

En el referido texto de Mols, hay un ejemplo que puede ayudar a dilucidar la potencialidad del enfoque. Se trata de una campaña de prevención del cáncer de piel en Australia que, utilizando eslóganes pegadizos y sencillos (Slip-slopslap, Slipping on a shirt, Slopping on sun lotion and slapping on a hat), buscó persuadir a la ciudadanía sobre la manera de concebir lo que era un comportamiento sensato respecto al sol. La publicidad se emitió durante el verano y en horarios con alta audiencia. Un estudio posterior, realizado en jóvenes, demostró que el impacto fue beneficioso, ya que la protección solar aumentó considerablemente y disminuyó el porcentaje medio global de piel expuesta al sol (Dobbinson et al., 2015). 
En el ámbito latinoamericano puede mencionarse un caso nicaragüense. Hacia 2006 Macours y Vakis analizaron en seis municipios del noroeste de Nicaragua, donde el nivel de pobreza era extremo, los programas de trasferencia condicionada (en los cuales se asigna a familias en situación de pobreza o con bajos recursos un dinero mensual condicionado a la observación de ciertas normas sanitarias — vacunarse - y educativas — asistir a la escuela — para los hijos menores de edad) y observaron que la interacción con líderes comunitarios incrementaba la inversión de los hogares en educación, nutrición y actividades de generación de ingresos, y además mejoraba las actitudes de la población con respecto a su futuro (Macours y Vakis, 2014).

Asimismo, campañas audiovisuales contra la violencia de género y el acoso sexual, como la liderada por ONU Mujeres en México, conocida como \#NoEsDeHombres, pueden ser ilustrativas y entrar en este enfoque. La mayoría de campañas sobre la violencia sexual se dirigen a mujeres y niñas, sin embargo, si se busca generar un cambio sociocultural, es indispensable promover una actitud en los hombres que no sea violenta o nociva. El objetivo de la campaña es prevenir el acoso y otras formas de violencia sexual contra las mujeres y niñas desmontando ciertas ideas que se ven como «normales» entre una gran parte de los hombres 5 .

En una línea que prioriza la autoconciencia del ciudadano sobre cómo desarrollar sus acciones para así darle mayor control sobre su comportamiento y mejorar su bienestar, se encuentra también el enfoque steer. Utilizando el término reflexividad para referirse al proceso cognitivo de tener conciencia del mundo en el que se vive y poder cambiar una situación, investigadores de la Royal Society for the Encouragement of Arts, Manufactures and Commerce (RSA) sostienen que pensar sobre cómo pensamos tiene un potencial transformador para las personas. Al igual que el enfoque think, esta perspectiva demanda un involucramiento activo de los sujetos, pero a diferencia de dicho enfoque, que se centra en el aprendizaje que ocurre en la deliberación, este se focaliza en el autocontrol (Grist, 2010).

La estrategia steer mostró su utilidad con grupos de policías (cuando se busca mejorar su relación con la sociedad), taxistas (para que ahorren combustible) o ciudadanía en general (para que lleven a cabo hábitos saludables y ecológicamente responsables). El caso de la Policía Metropolitana de Londres es ilustrativo. Primero se realizaron entrevistas con funcionarios específicos, luego se reclutó a un gran número de policías para participar en seminarios deliberativos y se les pidió que registraran sus experiencias para posteriormente compartirlas en un segundo encuentro. Entre los problemas que se identificaron, se encontró la necesidad de una devolución objetiva de parte de sus compañeros sobre su trabajo o lidiar con factores como el cansancio, la frustración y el aburrimiento. Con base en ello, los investigadores recomendaron promover una serie de pequeños cambios como reuniones semanales cortas

5. Ver: <http://mexico.unwomen.org/es/noticias-y-eventos/articulos/2017/04/noesdehombres> [Consulta: mayo de 2017]. 
Tabla 1. Herramientas políticas de cambio de comportamiento.

\begin{tabular}{|c|c|c|c|c|}
\hline $\begin{array}{l}\text { Herramientas } \\
\text { de cambio }\end{array}$ & ¿En qué consiste? & Procedencia teórica & $\begin{array}{l}\text { Cómo se articula con } \\
\text { cultura de la legalidad }\end{array}$ & Propuesta y ejemplos \\
\hline Nudge & $\begin{array}{l}\text { Pequeño empujón que } \\
\text { busca guiar el compor- } \\
\text { tamiento alterando la } \\
\text { arquitectura de decisión }\end{array}$ & $\begin{array}{l}\text { Paternalismo libertario } \\
\text { (Thaler y Sunstein) }\end{array}$ & $\begin{array}{l}\text { Usando heurística y } \\
\text { sesgos para modificar } \\
\text { creencias y preferen- } \\
\text { cias e incrementando } \\
\text { el atractivo para que } \\
\text { los sujetos se sumen } \\
\text { a acciones de suma } \\
\text { positiva }\end{array}$ & $\begin{array}{l}\text { Incentivar el cumpli- } \\
\text { miento fiscal mediante } \\
\text { distintos tipos de técni- } \\
\text { cas o sensibilizar sobre } \\
\text { el peligro de abusar de la } \\
\text { ingesta de determinados } \\
\text { alimentos mediante } \\
\text { etiquetas de colores (rojo } \\
\text { para indicar la cantidad } \\
\text { de grasa y azúcar y } \\
\text { verde para lo sano) }\end{array}$ \\
\hline Think & $\begin{array}{l}\text { Autoreflexión y configu- } \\
\text { ración de preferencias } \\
\text { que nacen del encuen- } \\
\text { tro con el otro. Efectos } \\
\text { cívicos de la delibera- } \\
\text { ción pública }\end{array}$ & $\begin{array}{l}\text { Democracia deliberativa } \\
\text { (John, et. al.) }\end{array}$ & $\begin{array}{l}\text { Desarrollo de actitudes } \\
\text { cívicas fomentando el } \\
\text { debate y la formación } \\
\text { de nuevas preferencias. } \\
\text { Focalizar en intereses } \\
\text { generales }\end{array}$ & $\begin{array}{l}\text { Crear espacios de deli- } \\
\text { beración y encuentro } \\
\text { tales como presupuestos } \\
\text { participativos, jurados } \\
\text { ciudadanos o asambleas } \\
\text { de debate }\end{array}$ \\
\hline $\begin{array}{l}\text { Social Identity } \\
\text { Steer }\end{array}$ & $\begin{array}{l}\text { Interiorización de nue- } \\
\text { vos entendimientos del } \\
\text { grupo de pertenencia } \\
\text { como parte de su propia } \\
\text { identidad }\end{array}$ & $\begin{array}{l}\text { Teoría de la identidad } \\
\text { social (Mols, Turner) }\end{array}$ & $\begin{array}{l}\text { Interiorización } \\
\text { de nuevas pautas }\end{array}$ & $\begin{array}{l}\text { Recurrir a los líderes } \\
\text { sociales para ejemplificar } \\
\text { comportamiento social- } \\
\text { mente deseable }\end{array}$ \\
\hline
\end{tabular}

Fuente: elaboración propia.

(de 20 minutos) entre oficiales para reflexionar sobre decisiones y hábitos y promover autoconciencia (Rowson y Lindley, 2012).

Con el fin de sistematizar, en la tabla 1 se resumen los principales aspectos de las propuestas con base en su definición y procedencia teórica; se esboza, asimismo, cómo se articulan con la cultura de la legalidad y se mencionan algunos ejemplos.

\section{Conclusiones}

El incumplimiento normativo se encuentra ampliamente extendido en América Latina y se acompaña de la sensación de que existe un orden que difícilmente se respeta. Esto constituye un obstáculo para el desarrollo, para el bienestar individual y general y para la confianza social ${ }^{6}$. No da igual ser peatón o ciclista en un lugar donde se obedecen las normas viales que en otro donde no; no da

6. Si confiar supone estar dispuestos a creer, a ponerse en las manos de otro y a sentirse seguro haciéndolo, el incumplimiento normativo no genera ni las certezas, ni la predictibilidad que un acto cognoscitivo como confiar demanda. La baja confianza predice baja cooperación social y, en consecuencia, débiles democracias con desarrollos insuficientes. Ver: Güemes, 2016. 
igual vivir en una ciudad con altos índices de contaminación ambiental que en otra donde se recicla, se controlan las emisiones de dióxido de carbono y los niveles de polución son bajos; no es lo mismo contratar un servicio o emprender un negocio en escenarios donde los trámites de apertura son complejos, el cumplimiento contractual bajo y la cadena de dádivas excesiva que en un lugar donde no lo es; no es lo mismo acceder, en condiciones de igualdad, a un servicio público al que se tiene derecho (salud, educación, transporte, agua potable) que tener que emprender un largo camino de sobornos y/o atajos informales, etcétera.

Tomando como punto de referencia los nocivos efectos sociales, económicos y políticos que tiene el incumplimiento normativo, hemos buscado claves para intentar entender el arraigo del incumplimiento, conectando dimensiones racionales y emocionales, contextos socioculturales y prácticas históricamente construidas. Para tal fin, identificamos y diferenciamos algunas de las perspectivas vigentes, desagregamos las ideas teóricas y normativas que las sustentan y señalamos las consecuencias prácticas que se deducen de las mismas para atacar el incumplimiento.

Este ejercicio analítico se complementó, en la segunda parte del trabajo, con la presentación de estrategias de gobernanza soft que ofrecen nuevos instrumentos que pueden ser útiles para el diseño y la implementación de políticas públicas. Asumir que el incumplimiento es multicausal (responde a cálculos racionales, pero también a percepciones sociales, a la vigencia de otros órdenes normativos informales y a la propia construcción de la identidad) complejiza a la vez que amplía el campo de acción en la búsqueda de remedios. En este marco, puede ser valioso aportar nuevas y creativas herramientas a los diseñadores de políticas y a quienes se encargan de combatir el incumplimiento.

En una lectura tradicional del derecho, la ley tiene por objeto moldear el comportamiento alterando los retornos que los individuos obtienen ante diferentes tipos de conductas. Lo que hemos intentado demostrar aquí es que esto no siempre funciona y que hay dimensiones que suelen escaparse en el análisis de partida. Es para estos huecos donde las nudges (asumiendo la presencia de carencias cognitivas en la definición del comportamiento humano), las herramientas deliberativas (que apuestan por el cambio de hábitos desde el razonamiento colectivo y grupal) y las propuestas de cambio identitario (que parten de un examen autoconsciente de la situación y de la relación entre la construcción de identidad y lo cultural) se vuelven herramientas complementarias a las tradicionales y prometedoras en la búsqueda de soluciones.

En un Estado de derecho las leyes son iguales para todos, sean gobernados o gobernantes. Pero no basta con estipularlo, es necesario que los principios que lo configuran, entre los que se encuentran el imperio de la ley, la división de poderes, la fiscalización de la Administración y la protección de derechos, libertades fundamentales y garantías jurídicas, se conviertan en valores compartidos por los miembros de una sociedad (Díaz, 1998). Evidentemente, esta visión compartida es el resultado de un largo proceso histórico que requiere de complejos cambios culturales y no es posible inculcarlo de un día para 
otro. Frente a esquemas hiperlegalistas (crear nuevas normas e instituciones fiscalizadoras) o propuestas voluntaristas (apelar al altruismo), las ideas aquí presentadas pretenden ofrecer algunas posibles vías sobre las cuales transitar para avanzar, lenta pero firmemente, hacia la deseada meta.

\section{Referencias bibliográficas}

Altman, David (2002). «Prospects for e-government in Latin America: satisfaction with democracy, social accountability, and direct democracy». International Review of Public Administration 7 (2), 5-20.

<https://doi.org/10.1080/12294659.2002.10805002>

ARIelY, Dan (2012). Por qué mentimos... en especial a nosotros mismos. Madrid: Ariel.

- (2014). Las trampas del deseo. Como controlar los impulsos irracionales que nos llevan al error. Madrid: Ariel.

BANCO Mundial (2015). World Development Report 2015: Mind society and behavior. Washington. DC: World Bank. <https://doi.org/10.1596/978-1-4648-0342-0>

- (2017). World Development Report 2017: Governance and the law. Washington DC: World Bank. $<$ https://doi.org/10.1596/978-1-4648-0950-7>.

Barrera-Osorio, Felipe; Bertrand, Marianne; Linden, Leigh y Pérez-Calle, Francisco (2011). «Improving the design of conditional transfer programs: Evidence from a randomized education experiment in Colombia». American Economic Journal: Applied Economics, 3 (2), 167-195. $<$ https://doi.org/10.1257/app.3.2.167>.

Bergman, Marcelo y Rosenkrantz, Carlos (2009). Confianza y derecho en América Latina. México: Fondo de Cultura Económica y Centro de Investigación y Docencia Económica.

Brugué, Quim; Feu, Jordi; Güemes, C. et. al, (2018). «Del fallo al colapso de las políticas públicas: una cuestión de confianza democrática». Revista de Sociologia e Politica, 26 (67), Sept.

CASTRO, Lucio y SCARTASCINI, Carlos (2014). El diablo está en los detalles: algunas lecciones para el diseño de politicas públicas. Banco Interamericano de Desarrollo, Resumen de Políticas del BID [en línea], 232, <http://www.iadb.org/es/investigacion-y-datos/detalles-de-publicacion,3169.html?pub_id=IDB-PB-232> [Consulta: julio 2016].

CoHEn, Joshua (1989). «Deliberation and democratic legitimacy». En: HAmLIN, Alan y PETTIT, Phillip (eds.). The Good Polity. Oxford: Basil Blackwell.

Díaz, Elías (1998). El Estado de derecho y sociedad democrática. Madrid: Taurus.

Dobbinson, Suzanne, VolKov, Angela y WaKefield, Melanie (2015). «Continued impact of SunSmart advertising on youth and adults». Behaviors American Journal of Preventive Medicine 49 (1), 20-9. <https://doi.org/10.1016/j.amepre.2015.01.011>.

ELSTER, Jon (1990). Tuercas y tornillos, una introducción a los conceptos básicos de las Ciencias Sociales. Madrid: Gedisa.

ESCALANTE, Fernando (2005). Ciudadanos imaginarios. Memorial de los afanes y desventuras de la virtud y apología del vicio triunfante en la República Mexicana: tratado de moral y virtud pública. México: Colegio de México. 
García Villegas, Mauricio (2010). «Individuos sin sociedades. Sobre la cultura del incumplimiento de reglas en América Latina». En: VILLORIA, Manuel y WeNCES, Isabel (eds.). Cultura de la legalidad. Instituciones, procesos y estructuras. Madrid: Catarata.

GRIST, Matt (2010). STEER Mastering our behavior through instinct, environment and reason. RSA Project [en línea], <https://www.thersa.org/globalassets/pdfs/reports/ rsa-social-brain_web-2.pdf> [Consulta: mayo 2017].

GoldFrAnK, Benjamín (2006). «Los procesos de 'presupuesto participativo’ en América Latina: éxito, fracaso y cambio». Revista de Ciencia Política, 26 (2), 3-28. <https://doi.org/10.4067/S0718-090X2006000200001>.

GÜEMES, Cecilia (2017). "¡Nudge en América Latina? Incidir en comportamiento individual, obtener resultados colectivos». Reforma y Democracia, junio. <http:// old.clad.org/portal/publicaciones-del-clad/revista-clad-reforma-democracia/ articulos/068-junio-2017/Guemes.pdf>.

HADAD, María Gisela; COMELl, María y PETZ, María Inés (2012). «De las asambleas barriales a las asambleas socio-ambientales: la construcción de nuevas subjetividades políticas. Argentina 2001-2011». Astrolabio, 9, 302-332.

John, Peter, Graham, Smith y Gerry, Stoker (2009). «Nudge nudge, think think: two strategies for changing civic behavior». The Political Quarterly, 80 (3), 361-70.

<https://doi.org/10.1111/j.1467-923X.2009.02001.x>.

- (2011). Nudge nudge, think think. Experimenting with the ways to change civic behavior. London: Bloomsbury. <https://doi.org/10.5040/9781849662284>.

LATINNO (2017). Base de datos de innovaciones democráticas en América Latina. WZB Berlin Social Science Center [en línea], <https://latinno.net/es/innovations> [Consulta: marzo 2017].

MaZAr, Nina, Amir, On y Ariely, Den (2008). «The dishonesty of honest people: A theory of self concept maintenance». Journal of Marketing Research. XLV, 633-644. $<$ https://doi.org/10.1509/jmkr.45.6.633>.

MACOURS, Karen y VAKIS, Reno (2014). «Changing households' investment behaviour through social interactions with local leaders: Evidence from a randomised transfer programme». The Economic Journal, 124, 607-633. <https://doi.org/10.1111/ecoj.12145>.

MockUS, Antanas (1999). «Cambio cultural voluntario hacia la paz». En: OsPINA, Héctor; Alvarado, Sara y López Moreno, Ligia (comps.). Educación para la paz. Una pedagogía para consolidar la democracia social y participativa. Bogotá: Cooperativa Editorial Magisterio.

- (2003). «Cultura ciudadana y comunicación». Revista La Tadeo, 68, 106-111.

- (2006). «Una visión de los logros y los retos de Bogotá, a partir de algunos conceptos de Jon Elster». En: CANTÉ, Freddy y MocKus, Antanas. Acción colectiva, racionalidad y compromisos previos. Bogotá: Universidad Nacional.

Mols, Frank; Haslman, Alexander; JetTen, Jolanda y StefFens, Niklas (2015). «Why a nudge is not enough: A social identity critique of governance by stealth». European Journal of Political Research, 54, 81-98. <https://doi.org/10.1111/1475-6765.12073>.

Nino, Carlos (1992). Un país al margen de la ley. Buenos Aires: Ariel.

NORTH, Douglass (1993). Instituciones, cambio institucional y desempeño económico. México: Fondo de Cultura Económica. 
O'Donnell, Guillermo (1999). «Polyarchies and the (un)rule of law in Latin America». En: MÉNDEZ, Juan, O’Donnell, Guillermo y PINHeIro, Sergio. (eds.). The un(rule) of law and the underprivileged in Latin America. Indiana: University of Notre Dame Press.

Persson, Anna, Rothstein, Bob y Teorell, Jan (2013). "Why anticorruption reforms fail-systemic corruption as a collective action problem». Governance: An International Journal of Policy, Administration, and Institutions, 26 (3), 449-471. <https://doi.org/10.1111/j.1468-0491.2012.01604.x>.

ROTHSTEIN, Bo (2000). "Trust, social dilemmas, and the strategic construction of collective memories». Journal of Theoretical Politics, 12, 477-501.

ROwSON, Jonathan y LiNDLEY, Emma (2012). «Reflexive coppers: Adaptive challenges in policing» [en línea], <https://www.thersa.org/globalassets/pdfs/reports/rsa-reflexive-coppers.pdf> [Consulta: abril 2017].

Schwartz, Janet, Mochon, Daniel, Wyper, Lauren, Maroba, Josiase, PATEl, Deepak y ArIELY, Dan (2014). «Healthier by precommitment». Psychological Science 25 (2), 538-546.

Sousa SANTOS, Boaventura de (1998). "Participatory budgeting in Porto Alegre: Toward a redistributive democracy». Politics and Society, 26 (4), 461-510.

Sunstein, Cass (2016). "Why some nudges are ineffective». Preliminary draft [en línea], <http://ssrn.com/abstract=2809658> [Consulta: julio de 2016].

- (2017). Paternalismo libertario. ¿Por qué un empujoncito? Barcelona: Herder.

ThaleR, Richard y SunSTEIN, Cass (2009). Un pequeño empujón (nudge). El impulso que necesitas para tomar las mejores decisiones en salud, dinero y felicidad. Madrid: Taurus.

Villoria, Manuel y JiménEZ, Fernando (2014). «Estado de derecho, cultura de la legalidad y buena gobernanza». En: WenCES, Isabel, Conde, Rosa y BonilLA, Adrián (eds.). Cultura de la legalidad en Iberoamérica: Desafios y experiencias. Costa Rica: Facultad Latinoamericana de Ciencias Sociales (FLACSO).

VILLORIA, Manuel y IZQUIERDO, Agustín (2015). Ética pública y buen gobierno regenerando la democracia y luchando contra la corrupción desde el servicio público. Madrid: Tecnos.

WenCES, Isabel y SAUCA, José María (2014). «Cultura de la legalidad: movimiento y proyecto». En: WenCES, Isabel, CONDE, Rosa y Bonilla, Adrián (eds.). Cultura de la legalidad en Iberoamérica: Desafios y experiencias. Costa Rica: Facultad Latinoamericana de Ciencias Sociales (FLACSO). 
